\title{
Quality-Oriented Search for Depression Portals
}

\author{
Thanh Tang ${ }^{1}$, David Hawking ${ }^{2}$, Ramesh Sankaranarayana ${ }^{1}$, \\ Kathleen M. Griffiths ${ }^{3}$, and Nick Craswell ${ }^{4}$ \\ 1 Department of Computer Science, Australian National University, \\ Canberra, Australia \\ ttintang@gmail.com, ramesh@cs.anu.edu.au \\ 2 Funnelback Pty Ltd, Canberra, Australia \\ david.hawking@acm.org \\ 3 Centre For Mental Health Research, Australian National University, \\ Canberra, Australia \\ kathy.griffiths@anu .edu.au \\ 4 Microsoft Research, Cambridge UK \\ nickcr@microsoft.com
}

\begin{abstract}
The problem of low-quality information on the Web is nowhere more important than in the domain of health, where unsound information and misleading advice can have serious consequences. The quality of health web sites can be rated by subject experts against evidence-based guidelines. We previously developed an automated quality rating technique (AQA) for depression websites and showed that it correlated 0.85 with such expert ratings.

In this paper, we use AQA to filter or rerank Google results returned in response to queries relating to depression. We compare this to an unrestricted quality-oriented (AQA based) focused crawl starting from an Open Directory category and a conventional crawl with manually constructed seedlist and inclusion rules. The results show that postprocessed Google outperforms other forms of search engine restricted to the domain of depressive illness on both relevance and quality 1
\end{abstract}

Keywords: Health portal search, Quality filtering of search results.

\section{Introduction}

Web-delivery of information and interventions relating to depression and other mental health issues helps overcome reluctance to seek help induced by stigma. Unfortunately, information on many depression web sites does not accord well with scientific evidence 3. For commercial, religious or other motives, sites may promote unproven or even harmful treatments while failing to mention alternatives which have been proven to be effective.

It is desirable that search engines covering depression (or other health) content should bias results in favour of sites providing quality information. In the present

${ }^{1}$ An extended version of this paper gives more detail of methods and reports results of follow-up investigations: http://david-hawking.net/pubs/tang_ecir09_full.pdf 
study, we examine whether a previously published automated method for rating depression website quality (AQA, 4]) can be used to improve the quality of depression search results.

Our first aim was to determine whether using AQA to filter or rerank results from a global search engine, Google, would improve the quality of results for queries relating to depression. Our second aim addressed much smaller scale search facilities typically associated with health information portals. Can an unrestricted focused crawler starting with an Open Directory depression category seed list and using AQA quality ratings outperform a manually defined portal search facility?

To the best of our knowledge, the present paper is the first to:

1. Evaluate the three principal techniques for constructing subject portal search services: manual seedlist and rules, focused crawling, and filtering/reranking of a general search engine. Our evaluation is extensive (100 queries) and uses human assessors to rate both relevance and quality of search results.

2. Show that the quality of health search results returned by a highly-performing general search engine (Google) can be improved by post-filtering or reranking, using automatically derived quality scores.

\section{Previous Work}

A number of previous studies (e.g. Ilic et al. [5] and Bin and Lun [1]) have compared the effectiveness of general search engines with that of medical-specific engines in locating medical information. Our own previous study [1] included relevance and quality evaluations of health search engines but none of those engines were based on focused crawling or on quality filtering.

Evidence-based medicine defines rigorous procedures 2 for systematically reviewing scientific studies, assessing the level of evidence they provide and, from them, synthesizing guidelines for clinical practice. Jorm et al 7,8 , have applied this approach in rating a large number of conventional and alternative treatments for depression.

One might expect that the static scores used by Web search engines would predict evidence-based site ratings, but we found [4 only a moderate correlation $(r=.61, p=0.002)$ between expert ratings and PageRanks reported by the Google toolbar (and then only after excluding PageRanks of zero). By contrast, we found a high correlation $(r=.85, p<0.001)$ between our AQA method (see below) and the expert ratings.

\section{The Present Study}

\subsection{Overview of the AQA Method}

The reader is referred to 4 for a description of the AQA method. In essence, a weighted relevance query is learned by contrasting term probabilities in a set 
of documents relevant to depression and a set with low probability of relevance. A quality query is learned using a similar method contrasting high quality documents with a set with low probability of being high quality. Pages are scored against these queries using the BM25 formula [9]. Site relevance and quality scores take into account both the number of pages on the site which match the applicable query to some degree, and the mean score of those pages.

\section{$3.2 \quad$ Engines}

Six "engines" were studied in this experiment. BPS and QFC used an implementation of the BM25 9] formula. GoogleD, FGD1 and FGD2 results rely on Google's proprietary algorithm. Note that Google no longer reports its index size.

\begin{tabular}{|c|c|c|}
\hline \multirow{3}{*}{$\begin{array}{l}\text { Engine } \\
\text { BPS }\end{array}$} & URL & Pages in index Notes \\
\hline & bluepages.anu.edu & $2.07 \times 10^{4}$ Depression specific \\
\hline & .au/search.html & \\
\hline GoogleD & google.com & $\begin{array}{c}\approx 10^{10} \text { Google with "depression" } \\
\text { added to queries }\end{array}$ \\
\hline FGD1 & not publicly available & $\begin{aligned} & \approx 10^{10} \text { Filtered out } 75 \% \text { of the } \\
& \text { bottom-quality sites from } \\
& \text { GoogleD results }\end{aligned}$ \\
\hline FGD2 & not publicly available & $\begin{aligned} \approx 10^{10} & \text { Filtered out } 95 \% \text { of the } \\
& \text { bottom-quality sites from } \\
& \text { GoogleD results }\end{aligned}$ \\
\hline $\mathrm{QFC}$ & not publicly available & $4.18 \times 10^{4}$ Quality focused crawler \\
\hline AvgRan & not publicly available & $\begin{array}{c}\approx 10^{10} \text { Averaging Google Rank } \\
\text { and quality rank }\end{array}$ \\
\hline
\end{tabular}

BluePages Search (BPS): BPS is fully described in [11. It is a crawled index controlled by a seed list of more than 200 sites plus corresponding URL-based include/exclude patterns. The seed list and patterns were manually constructed in 2004. Unfortunately, they had not been updated prior to the present experiments, due to heavy time cost and limited resources.

GoogleD: GoogleD converts Google into a depression-specific search facility by adding the word depression to queries which don't already include it. See [11. GoogleD results were obtained using the Google API. Coverage of GoogleD is very large.

Quality Focused Crawl (QFC): The QFC engine is as described in [12, but working with an enlarged seed set comprising 301 depression category sites taken from the Open Directory2. Its "frontier" of unvisited candidate pages is ordered by the product of estimated relevance and quality scores.

The QFC crawler was not engineered to the necessary degree, nor were machine and network resources available to support a crawl to even approach Google coverage. Accordingly, the QFC crawl was stopped after 41,823 pages, about

2 dmoz.org 
twice the size of BPS. At this stage, the crawler had already become trapped in a site with dynamically generated URLs.

Quality filtering of Google results(FGD1 and FGD2): FGD1, FGD2 and AvgRankGD (see below) rely on obtaining a deep results list from GoogleD and deriving a corresponding sorted site list as follows:

- The Google API was used to collect the top 1,000 ranked results for each of the 100 test queries. 97,445 distinct results were collected and organised according to the Google rank of each URL for each query, forming a list called here the Google ranked list.

- From the Google ranked lists aggregated across queries, a site list was derived using host name prefixes and other heuristics.

- The AQA procedure described in [4] was applied to the site list, and the sites were then sorted, resulting in a list of websites with quality scores arranged in descending order of quality, called here the sorted site list.

The Google ranked list was then filtered, selecting for each query the top ten URLs belonging to sites whose quality scores reached a threshold, while preserving their original Google rank order. We arbitrarily set two thresholds designed to include approximately the top $25 \%$ of sites (FGD1) and approximately the top $5 \%$ of sites (FGD2). This resulted in 360 and 58 sites respectively.

Quality re-ranking of Google results(AvgRankGD): Another engine, AvgRankGD, was created by combining the GoogleD rank and the rank of its site based on the quality score of that site. Each URL in the Google ranked list was re-ordered according to the mean of these two ranks. The ten top ranked URLs for each query were selected as the AvgRankGD result set.

\subsection{Query Set}

Queries were submitted to each primary engine and result sets (of ten results) obtained directly or after post-processing were pooled for each query and subjected to blind judging. Note that, effectively, the test corpus is the entire public Web, and the performance of a retrieval system depends upon its coverage as well as its ranking function.

We used the set of 100 queries used in [11. The query set comprised an equal mix of names of depression treatments (e.g. 'alcohol avoidance', 'cognitive behaviour therapy' and 'cipramil') and depression-related queries submitted to search engines. Depression treatment names were taken from a systematic review by Jorm et al. [7.

\subsection{Judgments}

Relevance was measured for the full set of 100 queries. Judges were asked to assign one of four levels of relevance using the Sormunen scheme [10]).

Quality was assessed only for 50 treatment queries. Restricting quality judgments to treatment queries allowed the use of non-expert judges. They were asked to judge: 
- Does this page recommend or support the treatment (positive), oppose the treatment (negative), or neither (neutral)?

Recommending an effective treatment, or recommending against an ineffective treatment, was considered correct advice. Recommending against effective or in favour of ineffective treatments was considered incorrect advice.

The treatments were classified on the basis of a systematic review of the effectiveness of depression treatments [7] into the categories listed in Table 2

A quality score for engines was derived by asking two subject experts to assign appropriate rewards and penalties to each combination of treatment value and recommendation as shown in Table 2. The experts assigned the rewards and penalties without knowledge of experimental results.

\section{Results}

\subsection{Relevance Results}

Significance tests were conducted using Wilcoxon Signed Rank tests with confidence level of $95 \%$.

Table 1 presents relevance performance of the six engines for two measures: mean modified average precision (MAP) 3 and mean normalised discounted cumulative gain (NDCG) [6]. GoogleD returned best results on both relevance measures, followed by FGD2, FGD1, AvgRankGD, QFC, and BPS. The results were almost consistent for both measures, except for an order swap between FGD1 and FGD2.

There was no significant difference in mean MAP scores between the best performer, GoogleD, and any of the Google variants. However, GoogleD significantly outperformed QFC $(p<0.001)$ and QFC outperformed BPS $(p<0.0001)$.

\subsection{Quality Results}

Table 3 lists the overall quality scores for each engine and the basis on which the scores were calculated. AvgRankGD achieved the highest overall quality score, followed by FGD2, QFC, FGD1, GoogleD, and BPS.

GoogleD returned a lot of correct advice but also the highest number of pages giving incorrect advice. BPS and GoogleD were the worst performers for this measure, achieving similar ratios of correct advice to all advice $(74 \%$ and $76 \%$ respectively).

The numbers listed in Table 3 are dependent on the relevance of search results. For example, GoogleD retrieves more relevant pages than BPS, so it naturally retrieves more pages with advice. Note also that the domain-specific engines that use AQA (noted as "Y" in the $A Q A$ column) all achieved better quality ratios than those without AQA.

${ }^{3}$ I.e. mean average precision as used in TREC, but where the denominator is set to the judging depth (here 10) when the number of known relevant documents is greater than that. 
Table 1. Relevance results for the search engines. $M A P$ refers to Modified Average Precision and $N D C G$ means Normalised Discounted Cumulative Gain.

\begin{tabular}{lcc}
\hline Engine & mean MAP mean NDCG \\
\hline GoogleD & 0.554 & 0.709 \\
BPS & 0.256 & 0.469 \\
QFC & 0.400 & 0.566 \\
FGD1 & 0.543 & 0.707 \\
FGD2 & 0.548 & 0.683 \\
AvgRankGD & 0.513 & 0.657 \\
\hline
\end{tabular}

Table 2. Quality Rating. Positive means that the treatment is recommended by the page being judged. Negative means that the treatment is recommended against.

\begin{tabular}{lcc}
\hline Treatment rating & Positive Negative \\
\hline Very strong evidence $(* * *)$ & 4 & -5 \\
Strong evidence $(* *)$ & 3 & -4 \\
Some evidence $(*)$ & 1 & -2 \\
No evidence $(-)$ & -1 & 0 \\
Not Effective $(\mathrm{X})$ & -5 & 4 \\
\hline
\end{tabular}

Table 3. Number of documents recommended for different treatment types. Effectiveness ratings (e.g. '***' and 'X') are defined in Table2 Quality scores are the sum of the expert-assigned rewards and penalties listed in that table. All documents in the pool were judged. Neutral judgments (no recommendation for or against the treatment) are not reported.

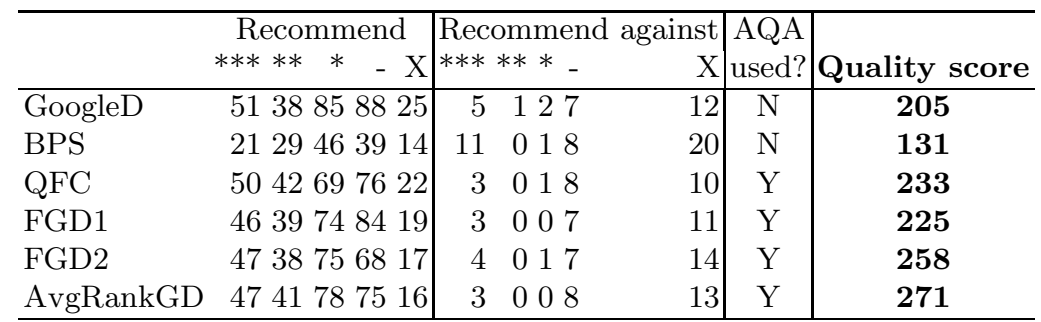

\subsection{Further Analysis of QFC Performance}

QFC retrieved significantly more relevant results than BPS, and also performed much better in terms of overall quality score as well as on overall proportion of good advice.

However, its quality result was not much better than that of GoogleD, and its relevance performance was worse. We hypothesize that QFC would have performed better if the crawl had been larger. The extended version of this paper (see footnote on page 1) describes our investigation of this hypothesis. 


\section{Discussion}

Our results suggest that if a global search engine operator wished to operate a vertical search portal within the domain of depression information, it could improve the quality of results returned by combining its normal ranking scores with quality scores derived from AQA. It could almost certainly do that more effectively than we could, because it has access to scores rather than ranks. We see no reason why anti-spam measures cannot be adapted to deal with any targeting of AQA scores by spammers. To maintain query response at reasonable levels, AQA ratings would have to be computed at crawl/index time rather than on the fly.

If a health organization wishing to operate a depression portal search were unable or unwilling to engage in a contractual arrangement with a global search provider, our results suggest that high quality, highly relevant results would be obtainable from a more robustly engineered quality-focused crawler starting from the Open Directory. Although actual results for QFC were poor relative to post-processed Google, our follow-up analyses suggest that the gap could be substantially narrowed with a more extensive crawl.

\section{Conclusions}

In this study, we have evaluated and compared representatives of three different techniques for constructing a domain specific search portal for the topic of depression: manual seedlist and rules, focused crawling, and filtering/reranking of a general search engine. Our evaluation considered not only the ability to retrieve relevant information, but the quality of the information retrieved, as measured against evidence-based guidelines.

We found that the best performance was obtained by pre-processing queries (adding the word depression) and filtering or re-ranking Google results using AQA scores. This approach achieved high relevance scores and returned pages with a high probability of providing correct advice. The rank averaging method appeared to outperform both filtering approaches on quality measures but was outperformed by them on relevance measures. Further work may suggest better combining methods than any of the three studied here.

Approaches available in the absence of cooperation with a major search provider failed to achieve the coverage of the Google variants. The apparent decline in relevance and quality scores for BPS since the initial study two years earlier (see extended version of this paper for more detail) almost certainly illustrates the effect of lack of maintenance. It highlights the amount of effort required to maintain a service based on manually defined seedlist and inclusion patterns.

Relevance and quality results for the quality-focused crawling approach, coupled with previous analysis of crawl progress, suggests that this approach to subject portal search is much more viable than manual definition. With additional engineering and a more extensive crawl, it is likely that relevance and quality scores could be improved over those reported here. 
An obvious direction for future work is to confirm that the AQA is capable of generalisation to other health topics. A study in the domain of obesity is currently under way.

\section{References}

1. Bin, L., Lun, K.: The retrieval effectiveness of medical information on the web. International Journal of Medical Informatics 62, 155-163 (2001)

2. Clarke, M., Oxman, A.: Cochrane Reviewers' handbook 4.1.1. The Cochrane Library, Oxford (2001)

3. Griffiths, K., Christensen, H.: Quality of web based information on treatment of depression: cross sectional survey. BMJ 321(7275), 1511-1515 (2000)

4. Griffiths, K., Tang, T., Hawking, D., Christensen, H.: Automated assessment of the quality of depression websites. Journal of Medical Internet Research 7(5) (2005), http://www.jmir.org/2005/5/e59/

5. Ilic, D., Bessell, T., Silagy, C., Green, S.: Specialized medical search-engines are no better than general search-engines in sourcing consumer information about androgen deficiency. Human Reproduction 18(3), 557-561 (2003)

6. Järvelin, K., Kekäläinen, J.: IR methods for retrieving highly relevant documents. In: Proc. SIGIR 2000, Athens, Greece, pp. 41-48 (2000)

7. Jorm, A., Christensen, H., Griffiths, K., Korten, A., Rodgers, B.: Help for depression: What works (and what doesn't). Centre for Mental Health Research, Canberra, Australia (2001)

8. Jorm, A., Christensen, H., Griffiths, K., Korten, A., Rodgers, B.: Effectiveness of complementary and self-help treatments for depression. Medical Journal of Australia 176, S84-S96 (2002)

9. Robertson, S.E., Walker, S., Hancock-Beaulieu, M., Gatford, M.: Okapi at TREC-3. In: Proc. TREC-3, November 1994, pp. 109-126. NIST spec. pub. 500-225 (1994)

10. Sormunen, E.: A method for measuring wide range performance of Boolean queries in full-text databases. Ph.D thesis, University of Tampere (2000), http://acta.uta.fi/teos.phtml?3786

11. Tang, T., Craswell, N., Hawking, D., Griffiths, K., Christensen, H.: Quality and relevance of domain-specific search: A case study in mental health. Information Retrieval 9(2), 207-225 (2006)

12. Tang, T., Hawking, D., Craswell, N., Griffiths, K.: Focused crawling for both relevance and quality of medical information. In: Proc. CIKM 2005, pp. 147-154 (2005) 